\title{
Structural Change and Social Governance of the Causes of Drug Abuse*
}

\section{-Analysis on In-Depth Interviews of 50 Individual Drug Abusers}

\author{
Shuyue Yan \\ Criminal Law Science, Beijing Normal University, Beijing, China \\ Email:na.jiang@bnu.edu.cn
}

How to cite this paper: Yan, S. Y. (2020) Structural Change and Social Governance of the Causes of Drug Abuse. Chinese Studies, 9, 42-52.

https://doi.org/10.4236/chnstd.2020.91004

Received: January 23, 2020

Accepted: February 24, 2020

Published: February 27, 2020

Copyright $\odot 2020$ by author(s) and Scientific Research Publishing Inc. This work is licensed under the Creative Commons Attribution International License (CC BY 4.0).

http://creativecommons.org/licenses/by/4.0/

\begin{abstract}
In China, it is a systematic program to deploy causal investigation on drug abusers, investigation on the cause for drug abuse involves an exploration of interdisciplinary, including law, sociology and psychology. Based on ethical norm, Beijing normal university project team deploys causal investigation on 50 drug abusers with stratified random sampling method, in search of structural change of the causes for drug abuse through interviews. There are new and nonnegligible reasons for drug abuse due to the change of social governance. Combined with analysis of reasons for drug abuse, targeted educational correction and return guidance should be deployed. Meanwhile, social governance on destigmatization and anti-discrimination should be strengthened to reinforce the effectiveness of drug addiction treatment.
\end{abstract}

\section{Keywords}

Drug Abuse, Structural Change, Social Variables, Self-Cognition

Drug abuse has become one of social issues that captured world attention, the most important measure to tackle drug abuse is preventing drug addiction. As long as controlling the emergence of new drug abusers, the number of drug abusers will naturally decrease. The reason for drug abuse is the starting point of analyzing drug abuse behaviour, therefore the reasons for drug abuse are the fundamental research of drug abuse behaviour study. In 2019, project team deployed interviews and investigation targeting at AIDS addicts under compulsory detoxification in S Province local drug rehabilitation centres (Z Centre, D Centre). Project team adopted stratified random sampling method to stratify sample *The project was funded by Sichuan Provincial Administration of Drug Rehabilitation. Based on privacy protection, the sensitive data involved has been treated technically. 
them according to nationalities, age, marital status, detoxification period (physiological detoxification period, rehabilitation period, regression period), causes of HIV infection etc. Z Centre and D Centre constituted the resource database of this investigation, from which 30 males and 20 females AIDS addicts under compulsory detoxification were selected to form two interview samples, with a total of 50 effective interview records recovered. In terms of information of rights, interview statements, questioning patterns and other aspects, the project team emphasizes the concept of "Respect" and "Empathic approach". After all, participants signing the informed participation consent individually, the project team conducted face-to-face structured interviews with drug addicts under compulsory detoxification. Through contacting with drug addicts, the author first asked the question "why did you take drugs from the very beginning" to drug addicts under compulsory detoxification. According to the authorization, the interview process of drug addicts under compulsory detoxification who signed and ticked "agree" to the recording was recorded, and their answers were collected and categorized to summarize the causes of drug abuse.

\section{Introduction of the Research Issue}

Drug control is essential to strengthen the innovation of social governance. According to 2018 Progress Report published by China National Narcotic Control Committee, in 2018, China uncovered 109,600 drug-related crimes, captured 137,400 suspects and seized 67.9 tons of various drugs; China investigated and penalized 717,000 person-time drug abusers, dealt with compulsory isolation for drug rehabilitation on 279,000 person-time drug abusers, and ordered treatment in community drug detoxification and rehabilitation to 242,000 person-time drug abusers. In other words, the situation of drug control and detoxification across the country is complex and austere ${ }^{1}$. In recent years, the situation of drug abuse has presented patterns of double erosion of traditional drugs and new drugs, an interweaving of drug addiction and drug manufacture, and cross-influence of the drug problem and other social problems. Drug abuse is widespread and tends to spread wider with complex causes, mainly in social variables, weak self-regulation ability, social pressure and a low sense of self-identity. The number of harmful drug abuse groups increases with each passing day, and the number of criminal drug offences surges, which results in grave social disaster. This investigation selected $S$ province drug rehabilitation centres ( $Z$ Centre, D Centre) as the research samples to study the causes of drug abuse, which is helpful to understand the law of drug abuse and to plan strategies of drug control and detoxification.

It is expected to find out a more comprehensive, objective and specific distribution status of reasons for drug abuse in the compulsory isolation centre for

\footnotetext{
${ }^{1}$ According to the report, by the end of 2015, there were 404,000 drug abusers in China (excluding those who found no relapse, the death toll and the number of departures after three years of abstinence), including methamphetamine abusers 1.35 million, accounting for 56.1 percent. Methamphetamine has replaced heroin to become "Number One Drug".
} 
drug rehabilitation. According to this, suggestions under more scientific social governance can be put forward.

\section{The Causes of Drug Abuse from the Traditional Perspective}

The consensus of law, psychology, sociology and other disciplines is that the causes of drug abuse are complex and diverse. From the traditional perspective, social cybernetics theory has obtained wider acceptance. In recent years, one of the theoretical bases of drug control is that human behaviour is socially constructed $^{2}$. The perspective of social culture places the individual outside the social structure, and it puts primary concerns on the dynamic impact of social interaction and social culture on the individual, believing that the individual in the society is the product of socialization. The following factors are often considered as important factors to study the reasons for drug abuse and are also the focus of educational correction.

\subsection{The Effect of Social Variables on Drug Abuse Behaviour}

Human behaviour is a process of learning to imitate and gradually stereotyped in the social context, in which there are both social factors that promote people to commit crimes or obey the law. When the social group with which they interact frequently has more influence on the individual's criminal behaviour than the law-abiding behaviour, and the illegal behaviour conforms to the group-specific code of conduct, the individual is likely to break the law3 (Xia, 2003).

The interview data shows that more than 30 per cent of the respondents were encouraged to take drugs by drug abusers around them, including taking drugs themselves after failing to resist the temptation in the process of dissuading their partners (Figure 1). When asked about their first experience with drugs, most responded, "watching a friend take drugs makes me want to give it a try". Most drug abusers begin their first drug use experience in life through the inducement, solicitation and temptation of drug-abuse friends, and then their self-control changes from passivity to active drug use, and they gradually break away from the normal track of society. During this process, the attitude of drug-abuse group members towards drugs will gradually change, from fear of being rejected by the group, fear of not fitting in, to habituation and active contact with more senior drug-abuse friends, relying on the so-called "Brothers (Ge men)" and "Sisters (Jie men)", and finally making drug use a deep-rooted addiction for a long time. In this condition, drug addiction is no longer just to feed their addiction, and it has become the link of

\footnotetext{
${ }^{2}$ Peter L. Berger, Thomas Luckmann, “The Social Construction of Reality: A Treatise in the Sociology of Knowledge", Society constructed facts and social facts are manufactured and expressed.

${ }^{3}$ American Psychologist Albert Bandura believed that human behaviour, especially complex human behaviour, was acquired postnatally. The "social cognitive theory" of Bundura focuses on the role of observational learning and self-regulation in triggering human behaviour and attaches importance to the interaction between human behaviour and the environment.
} 


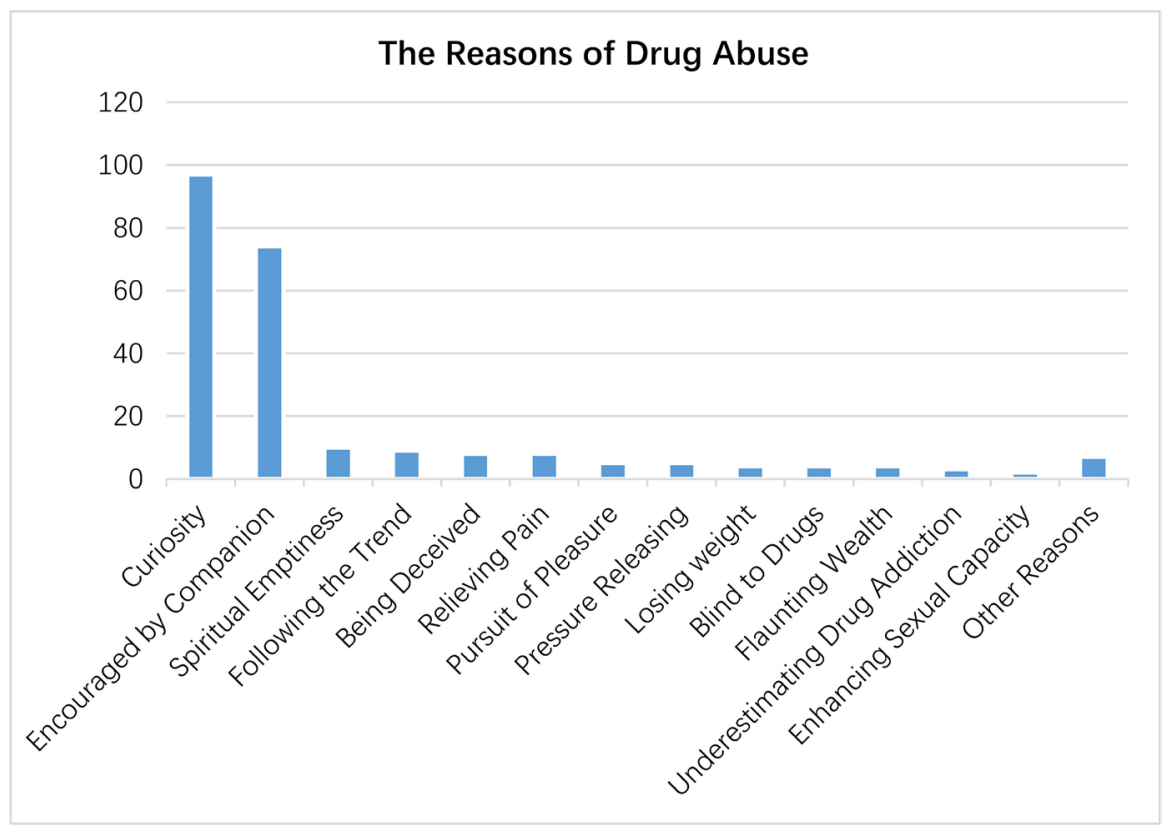

The data in the chart comes from the project of Fujian Provincial Educational Bureau: "Analysis of systematic Reasons for Drug Abuse" (Project No.: Ja02133s).

Figure 1. Statistics on the causes of drug abuse among 240 drug users in Fujian, 2002-2003. (Date source: Fujian education department project "Analysis of systematic Reasons for Drug Abuse”) (Project No.: Ja02133s).

"circle of drug-abuse friends".

\subsection{The Individuals' Weak Self-Regulation Ability}

The cognition of drug users to drug-taking behaviour plays a nonnegligible role in the drug-taking process. The weak self-regulation ability of individuals reflects the lack of self-control in the face of drug temptation, the inability to consider the consequences of drug use and the laissez-faire attitude toward harmful results.

Only when the individual has built up the psychology of rejecting drugs can he completely resist the temptation of drugs. In interviews, most people attributed their first drug use to "curiosity" (37 percent). Many scholars consider that one of the major reasons for drug abuse is people's curiosity about drugs, but it is only a superficial phenomenon. Everyone in the face of drugs as the new lure will have curiosity, but some can suppress while some with poor self-regulation ability choose to meet the "curiosity" (Figure 2).

Many drug users despise the harmful power of drug addiction, believing that they can control drugs and are easy to get rid of drug addiction. In the face of the temptation of drugs, which is a "novel toy", they cannot restrain their curiosity. Besides, due to their blind arrogance towards drugs, they believed that only taking a tentative and occasional attitude will not develop into uncontrollable and uncontrollable abuse. However, they fall into drug abuses after experimenting with drugs. 


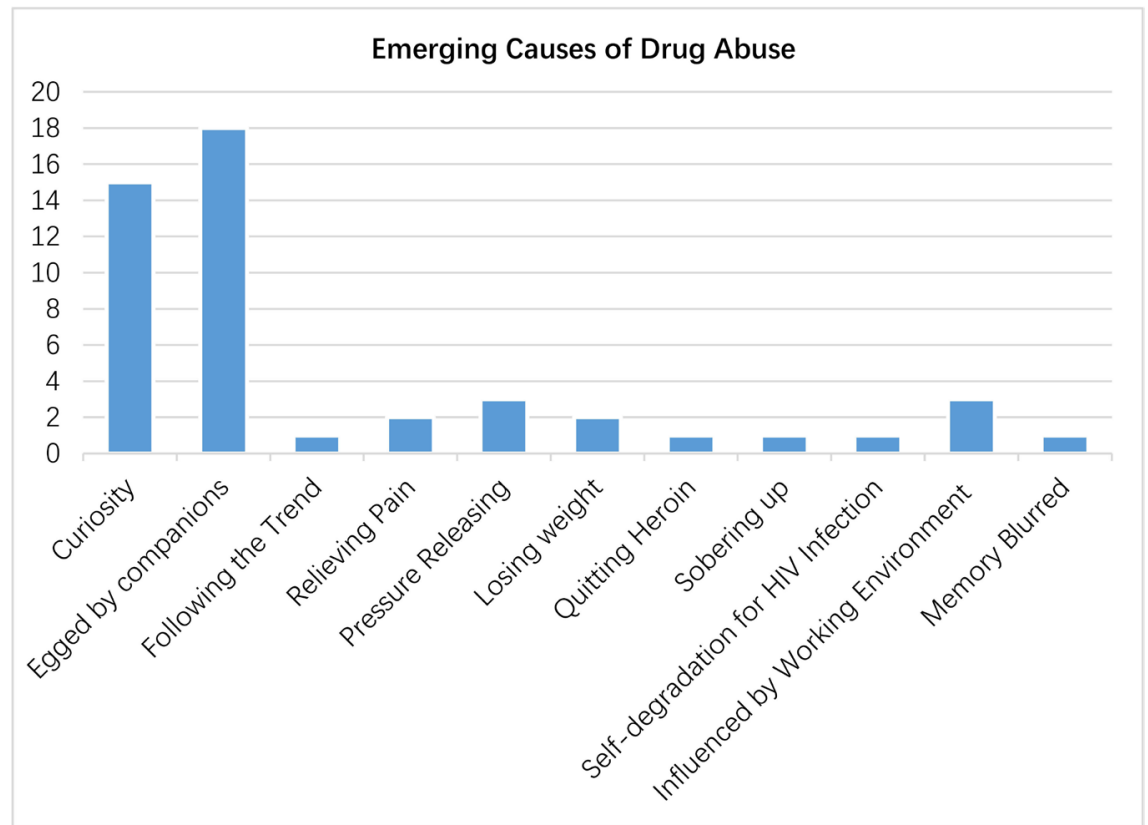

The data in the chart is from the survey of "Helping Experience of Centralised Management of AIDS Drug Addicts in Sichuan" (2019).

Figure 2. Statistics on the causes of drug abuse among AIDS Drug Addicts in Sichuan, 2019. (Date source: the survey of "Helping Experience of Centralised Management of AIDS Drug Addicts in Sichuan").

\subsection{Social Pressures Lead to Drug Use}

Merton believes that people at the bottom of society are the main source of the pressure of anomie because they lack access to success ${ }^{4}$. Social pressure leads to illegal drug use (Han, 2011).

According to the interviews, the main reason why some drug users take drugs for the first time and relapse many times is that they cannot effectively tackle social pressures. Their social relationship stress focuses on problems such as love and marriage frustration, emotional betrayal, social dependence and social fear. One gay drug addict pointed out that drug use can help him eliminate the loneliness brought by social interaction in a certain circle and relieve the inner pain. Reflecting on the reason for a relapse of drug abuse, some drug abusers pointed out the stress response caused by divorce. They lack internal or external social support, feel helpless and lost or even abandoned, in the thought of that they have no one to rely on, and they cannot adapt well in the face of society or adversity. It reflects the significant impact of social pressures brought about by marital management and emotional frustration.

${ }^{4}$ Merton's Anomie-stress theory: There will be a pressure of anomie when people cannot achieve their goals through legal means. People will take a series of means to alleviate pressure, in which lawbreaking and crimes are one of the means. An ideal society would be one in which all its members could use socially recognized means to achieve socially approved ends. However, in reality, society cannot always enable all members to achieve their ideal goals at all times and places in ordinary ways. As a result, some members may seek illegal ways to achieve their ideal goals. At the time, there are disconnection and conflict between socially advocated goals and socially approved means, then the anomie appears and the crime occurs. 
At present, the educational correction of drug abuse behaviour in China follows the overall scientific and objective cognition of the causes of drug abuse. However, structural changes have taken place in the structure of social hierarchy, groups and individual psychological cognition in China in many spheres. Some traditional subversive transformations have taken place in moral identity, right and wrong boundaries, and dangerous criticism of cognitive factors for specific things. Therefore, from the perspective of empirical investigation, it is worthy of research on which causes of drug abuse considered as the supporting factor of social governance.

\section{Unignorable Emerged Causes of Drug Abuse}

\subsection{Negative Selection after Learning of AIDS Infection}

Studies have shown that individuals experience anxiety, distress and other emotions when faced with problems beyond their control or when they are overwhelmed by stress. There was a significant negative correlation between self-anxiety and social support, and lower social support may lead to higher self-anxiety. It can be seen from the access degree of social support of AIDS patients that both subjective social support and objective social support will emerge subtly, but ultimately it depends on the utilization degree of support of AIDS patients.

Most AIDS patients lack positive awareness to use social support actively. Most AIDS patients after know oneself are infected with HIV, they generally have psychological problems such as severe anxiety, loss of self-esteem, fear of being abandoned, loss of self-identity, despair and extreme depression, resulting in reduced life cognition and attitude, which ultimately leads severe consequences of taking drugs, attacking others, self-harm, and even suicide (Lei et al., 2014).

2 percent of the drug addicts admitted to first starting to take drugs on testing positive for HIV. They confessed to having a self-defeating mentality, with some drug abusers on learning that they have AIDS saying they felt lost and their lives suffocated; some victims even chose to close themselves off from the outside world. According to the interview, the vast majority of AIDS patients can not choose the correct method to resolve their anxiety and loneliness after learning that they are infected with AIDS, which makes their life value deviate from the social moral standards, and some AIDS patients took drug abuse as a way to give up (Figure 2).

\subsection{Changes in Thinking Structure Caused by Social Interaction}

With the social turbulence and change, there are various types of cultures in today's society, and each culture has its own unique values and corresponding behaviours. Behaviours accepted by one group are likely to be alternative deviant behaviours for another group. Therefore, some drug addicts claim that they have not violated social norms, but just follow social norms and values that are dif- 
ferent from the mainstream. For them, drug abuse is only a form of leisure and entertainment, a means of entertainment and maintaining public relations without breaking laws.

With the rapid development of economic and social wealth, the reduced pressure to work for survival and increased spare time for leisure and entertainment, the pursuit of stimulation and enjoyment of social culture has become a social trend, providing the basis for the spreading of the drug. It is also on the rise as a leading cause of drug use (Figure 3). According to the interview, one of the male drug addict with AIDS admitted that he had gone to Macau to spend 40,000 yuan to keep a "mixed Vietnamese beauty". During this prostitution, he confessed to using methamphetamine together with prostitutes and having had sex eight or seven times without any security measures. Furthermore, he

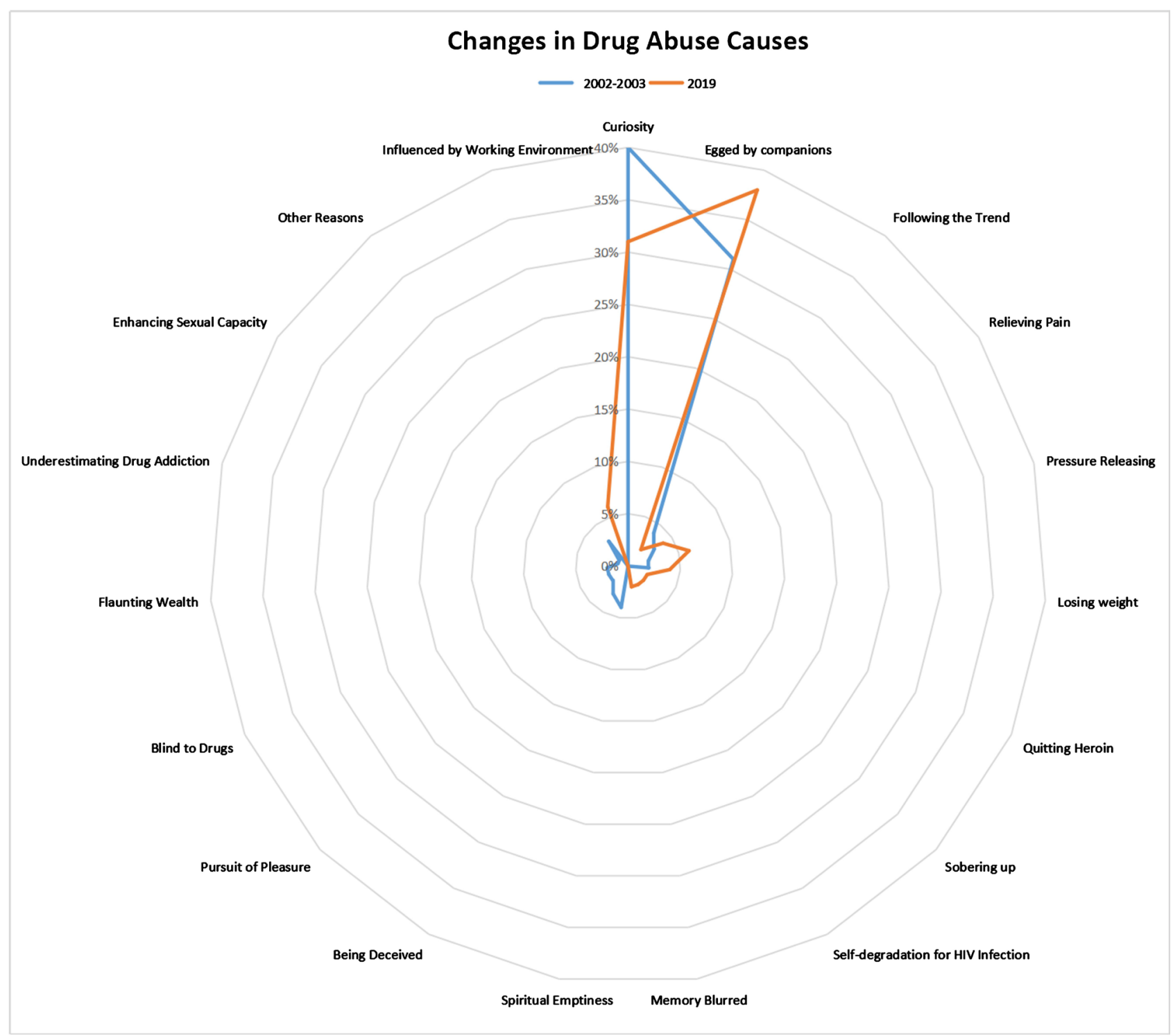

The data in the chart is from Figure 1 and Figure 2.

Figure 3. Changes in the causes of drug use. (Date source: Figure 1 and Figure 2). 
believed that this could not only allow people to cross borders to avoid the risk of breaking the law, but also to enjoy the pleasure and excitement of sexual liberation. Following are some records from interviewees:

"In fact, drug use is just as common as smoking, except that drug use is harmful to the body"

"It is ok to do drugs with my own money"

"In my circle of friends, drug use is common, just a means of social intercourse"

It can be seen from the interviewers' answers that they do not think that drug abuse is a severe problem. If they have sufficient funds to support the drug expenditure and do not steal or abduct, then they did not endanger social stability and safety.

\subsection{Wrong Choice Caused by Lack of Correct Awareness of Drugs}

Through the process of interaction with the external society, individuals form the cognition of the world and endow things with themselves understanding, which forms a set of values that in turn guide the individual's practical activities. Individuals obtain their new knowledge of drugs through interaction and communication with the surrounding environment. Therefore, in the process of initial exposure to drugs, drug users make trade-offs based on the constructed new understanding. Then, because of their weak knowledge of drugs and poor discernment ability, this group of people chose to use drugs.

Due to the nature of loving to beautiful, women, driven by the psychology of trying to lose weight quickly, are easily misled by some media and adopt terrible behaviours to lose weight. Taking drugs will have great impact on loss in appetite, physical excitement and insomnia, etc., which will lead to rapid weight loss. This superficial phenomenon has blinded many weight-loss people, thinking that drugs really work. A female drug addict said that at the beginning of her drug use, she reduced her weight from $65 \mathrm{~kg}$ to $50 \mathrm{~kg}$. Such a substantial change convinced her of the role of drug weight loss.

There are several cases in which drug users have talked about taking methamphetamine to get rid of heroin, believing that methamphetamine alleviates the abstinence reaction of heroin. Actually, they did not take heroin for a year after taking methamphetamine, while relapsed again into both heroin and methamphetamine soon. Methamphetamine is a stimulant, while heroin is a sedative. The excitement caused by methamphetamine and the sedation caused by heroin is exactly offset, thus giving the illusion that methamphetamine can cure heroin addiction.

\section{Suggestions for Social Governance}

\subsection{Develop Targeted Education for Specific Groups to Guide Correct Self-Cognition}

Drug addicts often have negative life cognition and attitude due to their own 
reasons (cognitive errors, personality defects), family environment (lack of continuity, emotional home education and personality home education), educational experience (low degree of literacy), social environment (excessive exposure to the dark aspect of society) and other different factors. Therefore, targeted education and correction should be carried out for different groups, so that they can learn to love themselves and respect others (Guo, 2000).

Addicts with AIDS have multiple roles: offenders, victims and patients. Offenders' misbehaviour labels, victims' socially disadvantaged status, and the near-incurable threat of HIV often leave this group in a state of conflict, which makes them unable to recognise themselves accurately. This self-confusion often leads this group to regard themselves like a broken jar broken falls the losers. Thus, when managing and assisting this group of victims, it is particularly important to pay attention to the education of their self-cognition and soundness of personality.

Aiming at the people who cause drug abuse due to lack of correct knowledge of drugs, the relevant departments should strengthen the propaganda of the adverse effects of drugs. For example, society needs to get weight loss groups to realise that drug use does not help people lose weight but can cost them their lives only. The rapid weight loss of drug users is a vicious reaction to drugs. The collapse of the human immune system, emotional instability, and disturbance of daily life caused by drug-using will lead to the inevitable thinness of drug users, which is a kind of pathological thinness. The correct view of weight loss is required among this group.

For different groups, different types of life education curriculum and systems for drug addicts under compulsory detoxification should be constructed. Cultivate their positive personality, enhance their positive experience, guide them to interact with the surrounding environment with a positive attitude and behaviour patterns, and enhance the value and meaning of life.

\subsection{Strengthen the Education of Destigmatisation, Anti-Discrimination and Ability to Cope with Social Pressure}

For most addicts, stigma and discrimination are essential factors that induce, reinforce and even lead to relapse. Influenced by some traditional views, lots of members of the public tend to define AIDS patients, sex workers, homosexuals or drug users as promiscuous or immoral people, believing that most of them are infected because they do not abide by social morality and public norms, and their life suffering serves them right $(\mathrm{Li}, 2012)$.

Faced with the fear and incomprehension of AIDS patients and homosexual groups, especially discrimination from society, they are incredibly vulnerable to get stress, anxiety, and moving towards drug use. It is still a complicated, lengthy and cumbersome process to eliminate the stigma and discrimination faced by people with AIDS. It is unrealistic to make a significant difference in a short period, which requires long-term unremitting and joint efforts of the whole society. Therefore, it is necessary to further strengthen the education of AIDS pa- 
tients, homosexuals and other minority groups, and guide them to adjust their mentality to deal with stigma and discrimination from the society. First of all, the inferiority complex that those minority groups may have had should be eliminated, and more positive psychological counselling is supposed to be provided to prevent them from internalising stigma and discrimination. Secondly, they should be encouraged to enhance interpersonal communication and interaction by participating in social organisations related to AIDS, drug addiction, homosexuality and other social activities. Finally, AIDS patients, homosexuals and their families should be encouraged to participate in destigmatisation and anti-discrimination activities actively. Besides, more attention is required for some stigma and discrimination cases to help protect their rights.

Z Centre once conducted a seminar and psychological counselling based on the psychological conditions of specific groups, especially the gay groups who are vulnerable to be harmed for the stigma and discrimination of homophobia. For instance, ten gay drug addicts under treatment mentioned that the lecture of "I Agree with Myself" had a high impact on them, which was conducive to preventing self-degradation and help them to be more confident when faced stigma and discrimination.

\subsection{Use the Support Function of the Protective Social Network to Conduct Regression Guidance and Consolidate the Effectiveness of Treatment}

Drug rehabilitation centres need to guide drug addicts to recognise that drug-using is deviant behavior and help them return to the mainstream. A vital indicator of the effectiveness of treatment for drug addicts is whether the patients who leave the rehabilitation centres can get rid of their addiction, recover physically and mentally, rebuild their confidence in life, and make a living. If these goals can be achieved, the treatment can be regarded as helping addicts return to society without risk to others. Otherwise, it is invalid regression and fails to reach the goal of abstinence education.

In order to promote the process that drug addicts under compulsory detoxification to restore their social functions, help them acquire social employment experience, and enhance their social adaptability, Z Centre organised the project of "employment experience" for drug detoxification personnel. This project is open to drug addicts whose remaining abstinence period is less than three months with relatively good abstinence effect and who apply for it by themselves. Besides, approvements and guarantees are required, not only from their family members but also the police in charge of them and the relevant department. Accompanied by the tutor of the police, the experiencers went to a shoe factory in $\mathrm{Z}$ city for the trail: six hours of labour per day, five days of labour per week, equal pay with ordinary workers. The experiencers worked in the factory during the day and returned at night to participate in drug treatment activities. They can also take part in social activities once a month, such as shopping, reading in the library, eating in the restaurant, visiting relatives on holidays, 
voluntary labour in welfare institutions and other social activities. According to the experience of $Z$ Centre, this project continues to this day. The integrity rate of the addicts who leave the drug rehabilitation centre after this project is higher than that of the personnel who return directly from the ordinary police team.

AIDS addicts face the same fear and despair when they learn of their HIV infection. They need to be understood and cared for, especially acceptance from their family members. Addicts could be encouraged to become pen pal or "letter lovers" for each other. For example, when a female drug addict left the drug rehabilitation centre, her pen pal from the $\mathrm{Z}$ police station accompanied her family to pick her up at the gate. According to the theory of externality, strengthening the harmless effect on the external can help to promote the communication between the drug addicts who have AIDS. Of course, there are still some disputes in practice.

\section{Conclusion}

This paper suggested that the causes of traditional drug use have changed, and more attention should be paid to various emerging factors. It is necessary to analyse the causes of multiple drug abuse in depth and carry out targeted prevention strategy based on the division of causes to reduce the deviation of social input and output. Accurately understanding the reasons for drug abuse is a necessary part of implementing accurate social corrections. At present, drug crimes are increasingly rampant. With the development of intelligent detoxification and the breakthrough of big data, the mastery of causality of drug abuse is still a mysterious and complicated sensitive field. In-depth understanding of drug abuse causes requires more critical interpersonal wisdom rather than technical ones.

\section{Conflicts of Interest}

The authors declare no conflicts of interest regarding the publication of this paper.

\section{References}

Guo, J. (2000). Prevention and Correction of Illegal Drug Use. Beijing: Law Press.

Han, D. (2011). A Sociological Study on Drug Abuse and AIDS: A Case Study of Drug Users in Jiangsu Province. Beijing: China Social Sciences Press.

Lei, X., et al. (2014). Guarding the Blue Lotus-The Centralized Management of Legal Care for AIDS Patients in China. Beijing: Law Press.

Li, W. (2012). Principles of Anti-Discrimination Law. Beijing: Law Press.

Xia, G. (2003). Research on Drug Banning from the Perspective of Sociology-Investigation on Adolescent Drug Abuse. Social Science, 10. 